\title{
Mapeando redes científicas multidisciplinares com WebQualis
}

Mapping multidisciplinary science networks in Brazilian Universities with WebQualis

Mapeando redes científicas multidisciplinares con WebQualis

Patrícia Dias dos Santos, mestre em Engenharia da Informação pela Universidade Federal do ABC. Endereço: Avenida dos Estados, 5001, bloco B, sala R801. CEP: 09210-580 Santo André, SP. Telefone: (11) 4996-0060. E-mail: patricia.santos@ufabc.edu.br.

Profa. Dra. Margarethe Born Steinberger-Elias, professora do Programa de Mestrado em Engenharia da Informação da Universidade Federal do ABC, doutora em Comunicação e Semiótica pela Pontifícia Universidade Católica de São Paulo. Endereço: Avenida dos Estados, 5001, bloco B, sala 943. CEP: 09210-580 Santo André, SP. Telefone: (11) 4996-0141. E-mail: mborn@ufabc. edu.br.

\section{Resumo}

Este trabalho faz parte de um estudo mais amplo sobre colaboração científica no sistema de universidades públicas brasileiras. Aqui o principal objetivo é caracterizar a adoção de práticas interdisciplinares no universo das universidades federais brasileiras. Tomando como referência o período de 1997 a 2007, uma primeira seleção de 8.948 artigos científicos foi extraída para análise, todos eles indexados no sistema WebQualis criado pela Capes (Coordenação de Aperfeiçoamento de Pessoal do Ensino Superior) e/ou indexados na Web of Science do International Institute for Scientific Information (ISI). Em seguida, foi selecionada uma amostra de 1.206 artigos, apontados nessas bases como inter ou multidisciplinares e escritos em coautoria por pesquisadores pertencentes a diferentes instituições. Usando indicadores bibliométricos e recursos da análise de redes sociais, foi 
possivel detectar e visualizar redes de colaboração interdisciplinar e, com base nesse conjunto de artigos, fazer um primeiro retrato das práticas de interdisciplinaridade no Brasil.

Palavras-chave: Comunicação Científica. Cienciometria. Redes Sociais. Colaboração. Interdisciplinaridade. Engenharia da Informação.

\section{Abstract}

This paper is part of a broader project dedicated to the study of scientific collaboration in the Brazilian public university system. The study's main objective is to characterize interdisciplinary practices in the universe of Brazilian federal universities. First a selection was made of 8,948 scientific journals published from 1997 to 2007 and indexed by the WebQualis System created by Capes (Coordenação de Aperfeiçoamento de Pessoal de Nivel Superior) and/or by the Web of Science developed by the International Institute for Scientific Information (ISI). Then a sample of 1.206 co-authored articles written by researchers belonging to different institutions was selected. These articles were identified by WebQualis or the Web of Science as being inter or multi-disciplinary in nature. Using bibliometric indicators and social network analysis, is was possible to detect and visualize interdisciplinary collaboration networks and, based upon the selected set of indexed articles, provide an initial picture of interdisciplinary practices in Brazil.

Keywords: Scientific Communication. Scientometrics. Social Networks. Collaboration. Interdisciplinarity. Information Engineering.

\section{Resumen}

Este trabajo hace parte de un estudio más amplio sobre colaboración científica en el sistema de universidades públicas brasileñas. Aquí el principal objetivo es caracterizar la adopción de prácticas interdisciplinares en el universo de las universidades federales brasileñas. Tomando como referencia el período de 1997 a 2007, una primera selección de 8.948 artículos científicos fue extraída para análisis, todos ellos indexados en el sistema WebQualis creado por Capes (Coordinación de Perfeccionamiento de Personal de la Enseñanza Superior) y/o indexados en Web of Science del 
International Institute for Scientific Information (ISI). A continuación, fue seleccionada una muestra de 1.206 artículos, apuntados en esas bases como inter o multidisciplinares y escritos en co-autoria por investigadores pertenecientes a diferentes instituciones. Usando indicadores bibliometricos y recursos del análisis de redes sociales, fue posible detectar y visualizar redes de colaboración interdisciplinar y, con base en ese conjunto de artículos, hacer un primero retracto de las prácticas de interdisciplinaridad en Brasil.

Palabras clave: Comunicación Científica. Cienciometria. Redes Sociales. Colaboración. Interdisciplinaridad. Ingeniería de la Información.

\section{Introdução}

A avaliação da excelência acadêmica de um pesquisador e da instituição à qual está vinculado comumente é medida por meio de critérios quantitativos. Ainda assim, já há um consenso de que tais critérios devem ser associados a outros que contemplem também a qualidade das publicações produzidas.

Meadows (1999, p. 89) sugere que "uma forma de avaliar a qualidade de uma publicação consiste em verificar o nível de interesse dos outros pela pesquisa”. Uma das formas mais simplificadas de se utilizar esse critério seria por meio da quantificação das citações que a pesquisa recebeu na bibliografia publicada posteriormente. Para recuperar essas informações, é muito utilizada a busca em bases de dados de citações categorizadas por periódicos. Grandes bases como a do Institute for Scientific Information (ISI) e o WebQualis da Capes tornaram-se essenciais para fornecer meios de dimensionar a produção científica. A indexação dos periódicos em bases de dados torna cada vez mais prático o acesso, para que a informação seja visualizada pela comunidade científica de forma rápida e sistemática.

Devido à complexidade crescente dos problemas científicos e societais, a colaboração científica interdisciplinar tem se tornado cada vez mais frequente e necessária. Os estudos bibliométricos ao longo das duas últimas décadas têm mostrado um crescimento contínuo do número de artigos publicados em coautoria entre representantes de disciplinas científicas diferentes, ligados a instituições e localidades geográficas distintas (KATZ e MARTIN, 1997; NEWMAN, 2001; MOODY, 
2004; SANCHO et al., 2006). Obviamente, as publicações em coautoria costumam ser mais citadas do que os artigos escritos por um único autor.

1. Conceituando a interdisciplinaridade na colaboração científica

Alguns programas de pesquisas são tão amplos, que estimulam novos conhecimentos em múltiplos campos. Um exemplo desse fenômeno é o Projeto do Genoma Humano. Às vezes, a criação de um novo campo ou disciplina é resultado da interação entre pesquisadores com um interesse prévio comum, como nos casos da Bioquímica, Ciência Cognitiva, Biologia Computacional e Nanociência. A pesquisa interdisciplinar também pode agregar valor a campos tradicionais. Pesquisadores em Nanociência, por exemplo, constroem pontes sobre diversas disciplinas e, ao mesmo tempo, usam a riqueza da sua experiência em Nanociência para abrir novas pesquisas e aplicações (BRUUN et al., 2005).

Uma das dificuldades em examinar a colaboração científica entre diversas instituições é a mortalidade de teorias que dizem respeito a novos modos colaborativos. Metáforas como a "Hélice Tripla”(LEYDESDORFF e ETZKOWITZ, 1996, 1998) fornecem um rótulo útil para novos modos colaborativos, mas pouca ajuda para distinguir entre eles. Mapear a interdisciplinaridade por meio da análise institucional é importante não só para criar e administrar oportunidades de colaborações científicas de larga escala, mas também para consolidar uma prática exercida pelas instituições de formar redes multiorganizacionais.

A colaboração é definida no contexto de cada trabalho de pesquisa e envolve diversos simbolismos e significados. Ela é frequentemente confundida com parcerias e uma variedade de redes de pesquisa formais e informais, alianças, pactos e cooperações. Tudo pode ser colaborativo, mas a finalidade e os resultados da colaboração devem ser considerados (KATZ e SMITH, 2000).

Refletindo sobre a colaboração interdisciplinar, Sonnenwald (2006) afirma que, como tópico de pesquisa, a colaboração científica é discutida em diversas disciplinas. De fato, em campos tão diversos como os da Comunicação Científica, Educação Científica, Psicologia do Conhecimento, Gestão da Ciência, Computação Científica, Sociologia da Ciência, Políticas Públicas em Ciência e Tecnologia ou 
Filosofia da Ciência, o tema da colaboração tem emergido em diferentes comunidades especializadas. Dependendo dos problemas selecionados para estudo, os métodos adotados são qualitativos, quantitativos ou mistos. Por exemplo, a Cienciometria estuda padrões de colaboração usando métodos quantitativos como estatísticas de coautoria, mas pode associar-se à Análise Linguística do Discurso Científico, que é predominantemente qualitativa.

\section{Metodologia}

Esta pesquisa faz parte de um trabalho mais amplo (SANTOS, 2010) sobre a produção científica colaborativa interdisciplinar de pesquisadores brasileiros. 0 presente artigo trata de autores filiados a universidades que pertencem ao sistema de Instituições Federais Brasileiras de Ensino Superior (Ifes). Foram tomados como referência artigos publicados em periódicos nacionais e internacionais no período de 1997 a 2007.

Os instrumentos utilizados para a coleta de dados foram bases de dados que representam importantes ferramentas para o registro da produção bibliográfica dos autores brasileiros. Para o levantamento da produção científica brasileira, utilizaram-se as revistas indexadas no Ano-Base (2007) do WebQualis, em consulta de periódicos por classificação/área de avaliação (Área de Avaliação Interdisciplinar) e, para o levantamento dos artigos publicados nessas revistas, utilizou-se a base de dados do ISI.

A primeira busca na base WebQualis do Portal da Capes gerou como retorno 13.178 periódicos avaliados em todas as áreas. Assim foi preciso criar uma série de filtros de forma a refinar a amostra e tornála mais compatível com o objetivo deste estudo. 0 primeiro deles foi a seleção de periódicos avaliados como interdisciplinar, o que gerou um total de 3.421 periódicos, ou seja, aproximadamente $26 \%$.

Após a identificação na base Qualis, realizou-se também uma busca no ISI para recuperar, no período compreendido entre 1997 e 2007, a totalidade dos artigos publicados em periódicos classificados nas duas bases como interdisciplinares. O objetivo foi identificar quais autores vinculados a que universidades federais haviam escrito em colaboração. Elaborou-se uma tabela dinâmica em Excel para facilitar a tabulação e compilação dos dados, utilizando campos da base WebQualis e da base ISI para a recuperação das informações. 
A etapa seguinte correspondeu à localização da produção científica nacional e internacional dos autores que compõem a amostra deste trabalho.

\section{Indicadores bibliométricos}

Neste trabalho, foram utilizados os seguintes indicadores de produção científica interdisciplinar:

1. Contagem do número de publicações: a partir do levantamento de artigos publicados em coautoria nos periódicos selecionados do ISI, foi possível fazer uma análise do número de artigos publicados em coautoria por região, instituição e área de conhecimento, sendo possivel assim mapear a colaboração interdisciplinar geograficamente por instituição, disciplina e área de conhecimento;

2. Participação percentual por região, instituição, disciplina e área de conhecimento: foi feita a partir dos resultados gerados pelo indicador de contagem do número de publicações;

3. Distribuição de produtividade de autores: a partir dos dados levantados, identificaram-se os autores responsáveis pelo maior número de publicações em coautoria por região, instituição, disciplina e área de conhecimento; e

4. Distribuição de revistas por disciplina: a partir da medição do número de artigos publicados em determinados periódicos, foi possível estimar o grau de relevância de periódicos em dada área do conhecimento e verificar que os periódicos que produzem o maior número de artigos sobre dado assunto formam um núcleo de periódicos, supostamente de maior qualidade ou relevância para aquela área.

\section{Instrumentos de análise estrutural de redes de pesquisa colaborativa interdisciplinar}

O ponto de partida para a análise das propriedades de uma rede social é calcular o número total de atores e o número possivel de laços e os que estão efetivamente presentes. Na análise de redes, serão empregadas as seguintes medições (HANNEMAN, 2001): 
1. Tamanho de uma rede: dado pelo total de atores que a compõem, é fundamental para a estrutura dos laços, pois os recursos e a capacidade que um ator tem para formar e manter laços são limitados: à medida que uma rede torna-se maior, o número de laços presentes diminui; acentuam-se as lacunas estruturais (BURT, 1992);

2. Quantidade de pares ordenados presentes na rede: depende do tipo de laço que há entre eles. No caso da rede de colaboração, em que os atores serão as universidades e as relações entre elas expressas pelas coautorias dos artigos produzidos, os laços são direcionais;

3. Distância: é a medida do intervalo entre determinado ator e os demais atores da rede. A forma de inserção dos atores na rede é mais complexa do que a simples análise de conexões diretas/adjacentes, pois dois atores podem estar indiretamente conectados. Atores que estão conectados por uma sequência curta de laços ou que têm maior número de conexões são mais estáveis e, portanto, apresentam maior previsibilidade de comportamento;

4. Densidade: essa medida é definida como a proporção de todos os laços que podem estar presentes do total de todos os possíveis e informa o nível de conexões diretas entre atores da rede;

5. Grau de intermediação: admite que um ator esteja em uma posição favorável se ele faz parte das conexões (distâncias geodésicas) que ligam outros pares de atores na rede e se desempenha o papel de intermediário das interações entre esses pares de atores. O grau de intermediação de um ator consiste na razão entre a soma de todos os passos geodésicos de todos os pares de atores da rede e o número de vezes que esse ator integra tais passos, multiplicado por 100. Esse índice registra o grau de afastamento de toda a rede em referência à rede estelar perfeita de igual tamanho; e

6. Conjunto Lambda (Lambda Set): classifica os laços existentes na rede de acordo com o fluxo que flui entre eles e mostra os conjuntos de atores que, se retirados, mais obstruiriam o fluxo entre todos os membros da rede. 


\section{Definição das amostras}

Os títulos utilizados neste estudo foram selecionados conforme as etapas a seguir, de modo a maximizar a possibilidade de que somente artigos interdisciplinares fossem analisados:

1. Forma de aquisição: foram considerados apenas periódicos classificados na Área de Avaliação Interdisciplinar do Qualis;

2. Forma de indexação: foram considerados apenas periódicos classificados na Área de Avaliação Interdisciplinar do Qualis e que fossem indexados pelo ISI;

3. Data de publicação: foram considerados apenas os documentos publicados no período de 1997 a 2007;

4. Endereço dos autores: foram considerados apenas os documentos em que pelo menos um coautor possuísse endereço de filiação no Brasil; e

5. Filiação dos autores: foram considerados apenas os documentos em que pelo menos um coautor fosse filiado a pelo menos uma das 55 universidades federais brasileiras que integravam o sistema das Ifes no período estudado.

\section{Resultados}

No ano de 1997, a base ISI continha um total de 7.952 periódicos indexados, dos quais 1.205 periódicos (15\%) foram classificados como multi ou interdisciplinar em 16 categorias (a distribuição de artigos e periódicos pode ser vista na Tabela 1). Da amostra inicial de 1.205 periódicos, $540(44,8 \%)$ apresentaram pelo menos um autor pertencente a uma das 55 Ifes. Foi coletado um total de 8.948 artigos, sendo que no período estudado houve uma produção mundial de 868.894 artigos e nacional de 18.420 artigos. Pôde-se então verificar que, de acordo com os parâmetros iniciais de seleção da amostra, a produção científica brasileira interdisciplinar representa um total de 2,12\% da produção mundial. Sendo assim, a amostra coletada representa $1,03 \%$ da produção de Ifes no âmbito internacional e 48,58\% no âmbito nacional. 
Tabela 1. Distribuição dos periódicos por área

\begin{tabular}{|c|c|c|c|c|c|c|c|c|}
\hline Categoria da Disciplina & $\begin{array}{l}\text { \# de } \\
\text { periódicos } \\
\text { indexados }\end{array}$ & $\begin{array}{c}\text { \# de } \\
\text { periódicos } \\
\text { analisa- } \\
\text { dos }\end{array}$ & $\begin{array}{c}\% \text { de } \\
\text { periódicos } \\
\text { analisa- } \\
\text { dos }\end{array}$ & $\begin{array}{c}\text { \# de } \\
\text { artigos } \\
\text { (1997- } \\
\text { 2007) } \\
\text { em escala } \\
\text { mundial }\end{array}$ & $\begin{array}{c}\text { \# de } \\
\text { artigos } \\
\text { (1997- } \\
\text { 2007) } \\
\text { em escala } \\
\text { nacional }\end{array}$ & $\begin{array}{c}\% \text { artigos } \\
\text { brasileiros } \\
\text { em escala } \\
\text { mundial }\end{array}$ & $\begin{array}{c}\text { \#de } \\
\text { artigos } \\
\text { (1997- } \\
\text { 2007) } \\
\text { publicados } \\
\text { - Ifes }\end{array}$ & $\begin{array}{c}\% \\
\text { artigos } \\
\text { de Ifes } \\
\text { em escala } \\
\text { nacional }\end{array}$ \\
\hline \multicolumn{9}{|l|}{ Ciências Biológicas, da Agricultura } \\
\hline \& Ambientais - Multidisciplinar & 30 & 11 & $36,67 \%$ & 29900 & 248 & $0,83 \%$ & 108 & $43,55 \%$ \\
\hline Agricultura - Multidisciplinar & 44 & 20 & $45,45 \%$ & 25497 & 3195 & $12,53 \%$ & 1266 & $39,62 \%$ \\
\hline Química - Multidisciplinar & 144 & 73 & $50,69 \%$ & 155997 & 3523 & $2,26 \%$ & 2098 & $59,55 \%$ \\
\hline \multicolumn{9}{|l|}{ Ciência da Computação, Aplicações } \\
\hline Interdisciplinares & 96 & 62 & $64,58 \%$ & 46500 & 817 & $1,76 \%$ & 416 & $50,92 \%$ \\
\hline Engenharia - Multidisciplinar & 81 & 40 & $49,38 \%$ & 4400 & 488 & $11,09 \%$ & 261 & $53,48 \%$ \\
\hline Geosciências - Multidisciplinar & 153 & 81 & $52,94 \%$ & 68614 & 1444 & $2,10 \%$ & 798 & $55,26 \%$ \\
\hline Humanidades - Multidisciplinar & 88 & 8 & $9,09 \%$ & 2780 & 70 & $2,52 \%$ & 31 & $44,29 \%$ \\
\hline Ciências da Vida - Multidisciplinar & 52 & 38 & $73,08 \%$ & 99638 & 799 & $0,80 \%$ & 317 & $39,67 \%$ \\
\hline Ciências dos Materiais - Multidisciplar & 200 & 62 & $31,00 \%$ & 142845 & 1576 & $1,10 \%$ & 784 & $49,75 \%$ \\
\hline Matemática Aplicações Interdisciplinares & 78 & 39 & $50,00 \%$ & 29758 & 722 & $2,43 \%$ & 395 & $54,71 \%$ \\
\hline \multicolumn{9}{|l|}{ Ciências Físicas, Químicas \& da Terra - } \\
\hline Multidisciplinar & 34 & 19 & $55,88 \%$ & 47792 & 790 & $1,65 \%$ & 412 & $52,15 \%$ \\
\hline Psicologia - Multidisciplinar & 108 & 30 & $27,78 \%$ & 15585 & 170 & $1,09 \%$ & 112 & $65,88 \%$ \\
\hline Ciências Multidisciplinares & 69 & 21 & $30,43 \%$ & 69861 & 784 & $1,12 \%$ & 434 & $55,36 \%$ \\
\hline Ciências Sociais, Interdisciplinar & 65 & 30 & $46,15 \%$ & 6310 & 185 & $2,93 \%$ & 89 & $48,11 \%$ \\
\hline Física - Multidisciplinar & 87 & 45 & $51,72 \%$ & 123417 & 3609 & $2,92 \%$ & 1720 & $47,66 \%$ \\
\hline Total & 1.205 & 540 & $44,81 \%$ & 868.894 & 18.420 & $2,12 \%$ & 8.948 & $48,58 \%$ \\
\hline
\end{tabular}

Segundo informações extraídas do sistema GeoCapes, na distribuição de Programas de Pós-Graduação por Status Jurídico no ano de $2007,53,1 \%$ dos programas são oferecidos por instituições federais, o que pode ter correlação com o fato de $48,58 \%$ das produções serem de Ifes, uma vez que grande parte da produção científica é resultado de pesquisas feitas por docentes/orientadores e estudantes de pósgraduação.

O Gráfico 1 mostra a distribuição de artigos publicados por Ifes. Observando-se o gráfico, é possível notar uma predominância de universidades das regiões Sul e Sudeste, com a UFRJ sendo responsável por $18,64 \%$ da produção nacional. Complementando as informações apresentadas no Gráfico 1, na Figura 1 essa disparidade de produção entre regiões pode ser melhor visualizada: as Ifes da região Sudeste são responsáveis por cerca de $48 \%$ da produção nacional, as regiões Sul e Nordeste por $22 \%$ e $20 \%$, respectivamente, e as regiões Norte e CentroOeste somam juntas $10 \%$ da produção. 


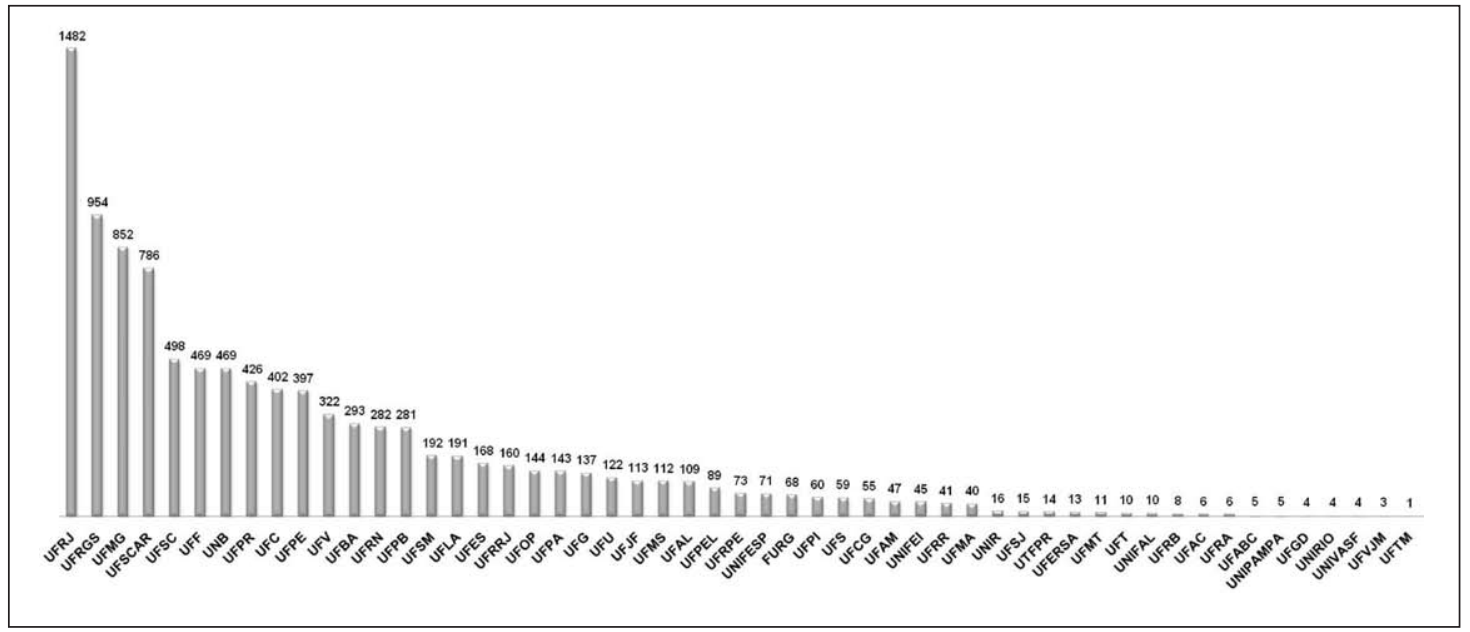

Gráfico 1. Distribuição de artigos científicos pelas Ifes no período 1997-2007

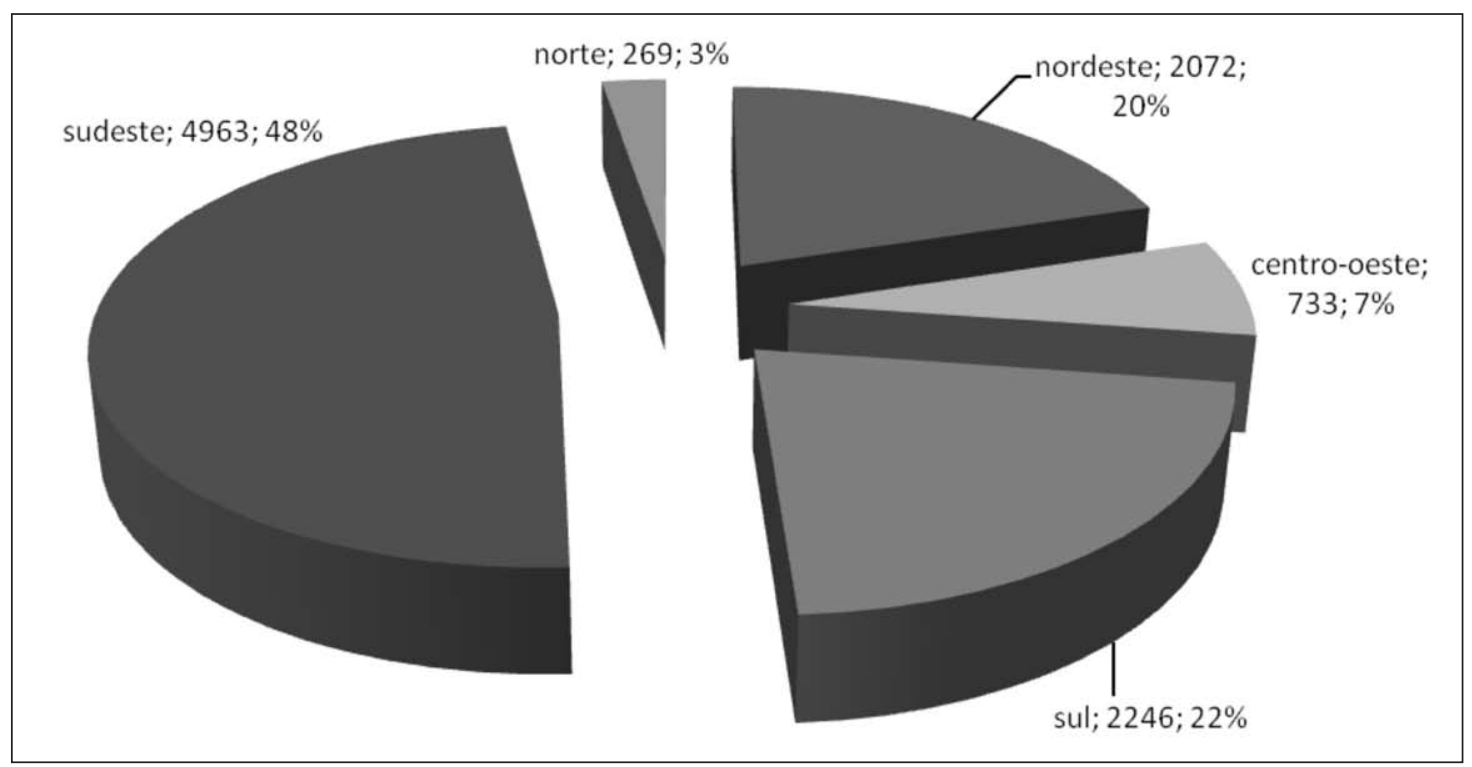

Figura 1. Distribuição de artigos científicos publicados pelas Ifes por região no período 1997-2007

No Gráfico 2, vê-se a dispersão do número anual de itens publicados no período, bem como uma curva ajustada pelo método dos mínimos quadrados como sugestão de inferência de padrão de comportamento.

Dada a função exponencial:

$$
y_{n}=y_{0} \cdot(1+r)^{n}
$$


A partir de (1), segue-se:

$r=\left(y_{n} / y_{0}\right)^{1 / n} \quad 1$

Uma fórmula alternativa a (1) é:

$y_{n}=y_{0} \cdot e^{b . n}$

A curva sugerida no Gráfico 2 tem um bom ajuste $(R 2=0,9512)$ e é descrita pela seguinte função:

$$
\mathrm{n}=402,99 . \mathrm{e}^{0,107 \text { (ano }} \text { 1997) (4) }
$$

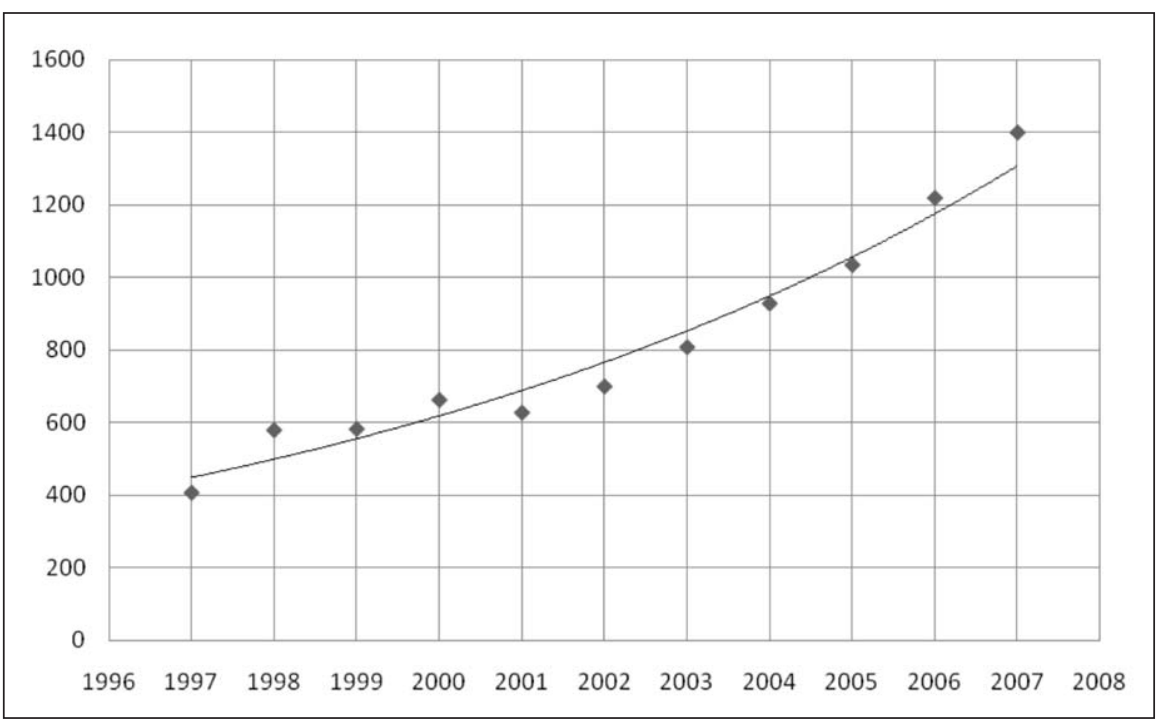

Gráfico 2. Gráfico de dispersão do número de artigos publicados pelas Ifes pelo ano de publicação, período de 1997 a 2007, e curva ajustada aos dados

Os parâmetros indicam respectivamente:

- 402,99: o número de artigos publicados começa com cerca de 403 artigos por ano;

- 0,107: vem crescendo geometricamente a um ritmo médio de $10,7 \%$ ao ano; e

- 1997: a data inicial é 1997, ou seja, inicia-se a contagem de tempo nesse ano (to=1997).

Apesar de essa curva mostrar um crescimento acentuado no período de 2001 a 2007, é preciso ter certo cuidado ao atribuir as razões 
desse crescimento exclusivamente ao aumento da produção científica nacional. Outros fatores também devem ser considerados, tais como: o aumento do número de periódicos indexados pela base ao longo desse período e o aumento da criação e/ou inserção de periódicos em meio digital, o que facilita o acesso e a recuperação da produção, por exemplo.

A Tabela 2 mostra a distribuição de artigos publicados nas 15 categorias estudadas no período 1997-2007, de acordo com o número de autores colaboradores por artigo. Observando-se a tabela, é possivel perceber que nas áreas de Física (Multidisciplinar) e de Ciências Multidisciplinares houve um número considerável de artigos que tiveram entre 100 e 600 colaboradores, o que pode indicar a participação de algum autor brasileiro em grandes grupos de pesquisa internacionais.

No caso da área de Física, isso ocorreu principalmente com pesquisadores que trabalham com pesquisas na European Organization for Nuclear Research(CERN) ou no Fermi National Accelerator Laboratory (Fermilab). Já em periódicos como Nature e Science, todos os artigos tiveram um número grande de coautores, o que também pode indicar que esses artigos resultaram de trabalhos realizados por grandes grupos de pesquisa internacional. Nas demais áreas, a distribuição de autores manteve-se predominantemente entre um e cinco por artigo, com exceção da área de Humanidades (Multidisciplinar), em que não houve nenhum artigo publicado em colaboração dentro da amostra estudada. Isso talvez se deva à característica de pesquisa de cada área. Na área de Humanidades pode prevalecer um caráter mais local, enquanto que a Física, Química ou Ciências da Vida, por serem áreas que requerem grandes investimentos em instalações, tendem a mobilizar profissionais e recursos de países diversos em projetos de grande porte. 
Tabela 2. Distribuição do número de autores por artigo das Ifes de acordo com a categoria no período 1997-2007

\begin{tabular}{|c|c|c|c|c|c|c|c|c|}
\hline Categoria da Disciplina & 1 autor & $\begin{array}{l}2-5 \\
\text { autores }\end{array}$ & $\begin{array}{l}6-10 \\
\text { autores }\end{array}$ & $\begin{array}{l}11-50 \\
\text { autores }\end{array}$ & $\begin{array}{l}51-100 \\
\text { autores }\end{array}$ & $\begin{array}{r}101-200 \\
\text { autores }\end{array}$ & $\begin{array}{c}201-500 \\
\text { autores }\end{array}$ & $\begin{array}{l}\text { mais de } \\
500 \\
\text { autores }\end{array}$ \\
\hline \multicolumn{9}{|l|}{ Ciências Biológicas, da Agricultura \& } \\
\hline Ambientais - Multidisciplinar & 7 & 58 & 27 & 9 & 3 & 3 & 1 & 0 \\
\hline Agricultura - Multidisciplinar & 16 & 973 & 272 & 5 & 0 & 0 & 0 & 0 \\
\hline Química - Multidisciplinar & 97 & 1480 & 500 & 21 & 0 & 0 & 0 & 0 \\
\hline \multicolumn{9}{|l|}{ Ciência da Computação, } \\
\hline Aplicações Interdisciplinares & 33 & 360 & 20 & 3 & 0 & 0 & 0 & 0 \\
\hline Engenharia - Multidisciplinar & 17 & 236 & 7 & 1 & 0 & 0 & 0 & 0 \\
\hline Geosciências - Multidisciplinar & 31 & 639 & 121 & 7 & 0 & 0 & 0 & 0 \\
\hline Humanidades - Multidisciplinar & 29 & 2 & 0 & 0 & 0 & 0 & 0 & 0 \\
\hline Ciências da Vida - Multidisciplinar & 11 & 201 & 73 & 24 & 4 & 3 & 1 & 0 \\
\hline \multicolumn{9}{|l|}{ Ciências dos Materiais - } \\
\hline Multidisciplar & 16 & 534 & 171 & 17 & 1 & 1 & 0 & 0 \\
\hline \multicolumn{9}{|l|}{ Matemática - Aplicações } \\
\hline Interdisciplinares & 31 & 351 & 13 & 0 & 0 & 0 & 0 & 0 \\
\hline \multicolumn{9}{|l|}{ Ciências Físicas, Químicas \& da } \\
\hline Terra - Multidisciplinar & 31 & 301 & 63 & 11 & 2 & 3 & 1 & 0 \\
\hline Psicologia - Multidisciplinar & 12 & 68 & 32 & 0 & 0 & 0 & 0 & 0 \\
\hline Ciências Multidisciplinares & 34 & 285 & 83 & 24 & 4 & 3 & 1 & 0 \\
\hline Ciências Sociais, Interdisciplinar & 64 & 25 & 0 & 0 & 0 & 0 & 0 & 0 \\
\hline Física - Multidisciplinar & 172 & 1312 & 112 & 23 & 2 & 11 & 21 & 67 \\
\hline Total de artigos & 601 & 6825 & 1494 & 145 & 16 & 24 & 25 & 67 \\
\hline
\end{tabular}

Conforme já dito anteriormente, o tamanho de uma rede social é dado pela quantidade de nós que a estrutura possui. A rede de Ifes em 2007 possuía 55 universidades. Para obter a densidade, os dados foram dicotomizados, de forma a indicar a presença ou não de colaboração entre duas ou mais universidades. Foi verificado dessa forma que o número máximo de Ifes colaborando em um dado artigo foi quatro, sendo que dos 8.948 artigos estudados houve colaboração entre duas ou mais Ifes em 1.201 deles, ou seja, aproximadamente 13\%. Assim, a densidade da rede estudada foi de 0,98\%.

A visão geral da rede colaborativa de Ifes pode ser vista na Figura 2. Observando-se a Figura 2, é possivel perceber que há dois nós isolados que correspondem à UFCSPA e Unifap, que não tiveram nenhuma publicação em colaboração. Os laços mais escuros entre dois nós correspondem às Ifes, que tiveram mais artigos publicados em 
colaboração, ou seja, que têm uma relação de colaboração mais forte em relação às demais e é possivel perceber que a proximidade geográfica é um fator importante observando que a UFMG e UFV, UFMG e UFOP e UFRJ e UFF tiveram os laços mais fortes.

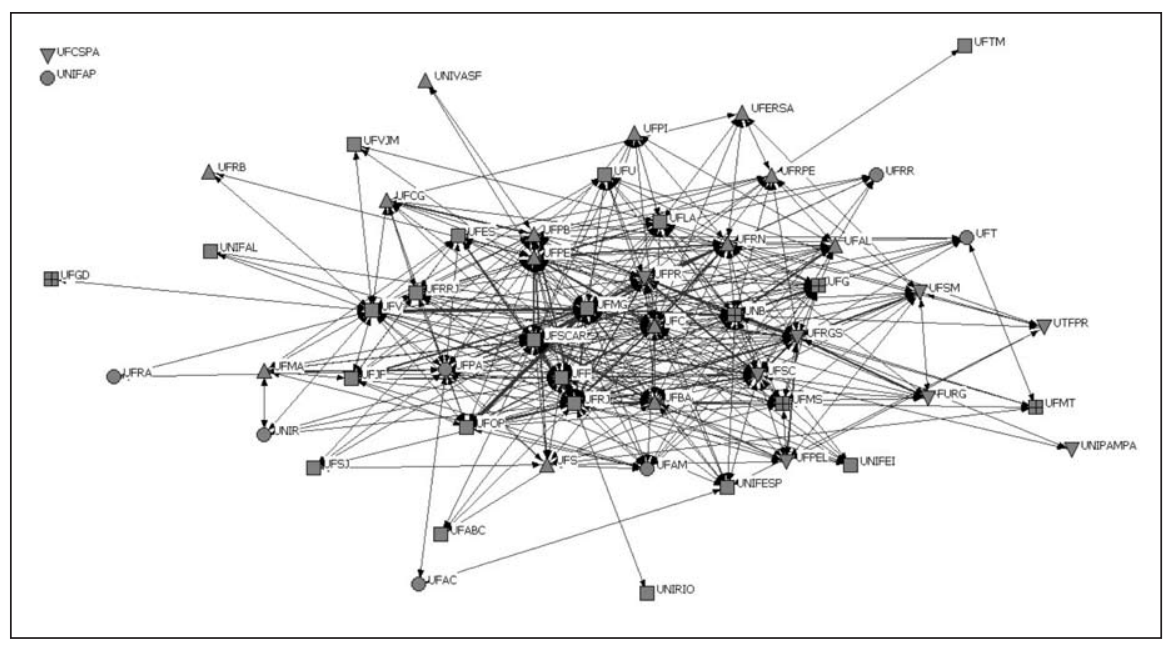

Figura 2. Visão geral da rede colaborativa de Ifes

A representação gráfica da análise de cluster para a rede (ver Figura 3) põe à mostra, no primeiro nível de agregação (nível 177), o agrupamento constituído por UFRJ e UFMG. 0 agrupamento seguinte (nível 156) incorpora UFSCAR, tendo em seguida UFF e UFRGS (nível 133). Essa representação gráfica mostra de forma hierárquica o nivel de agregação dos componentes, partindo daqueles que têm um maior fator de agregação (UFRJ e UFMG) e mostrando como os demais nós se agrupam em subniveis de importância na rede, de acordo com o número de relações (artigos em colaboração) mantidas com os demais nós. É importante notar que os nós correspondentes à UFCSPA e à Unifap têm um nivel de agrupamento igual a zero, pelo fato de não possuírem nenhum laço (colaboração) conectando-os com um outro nó da rede. 


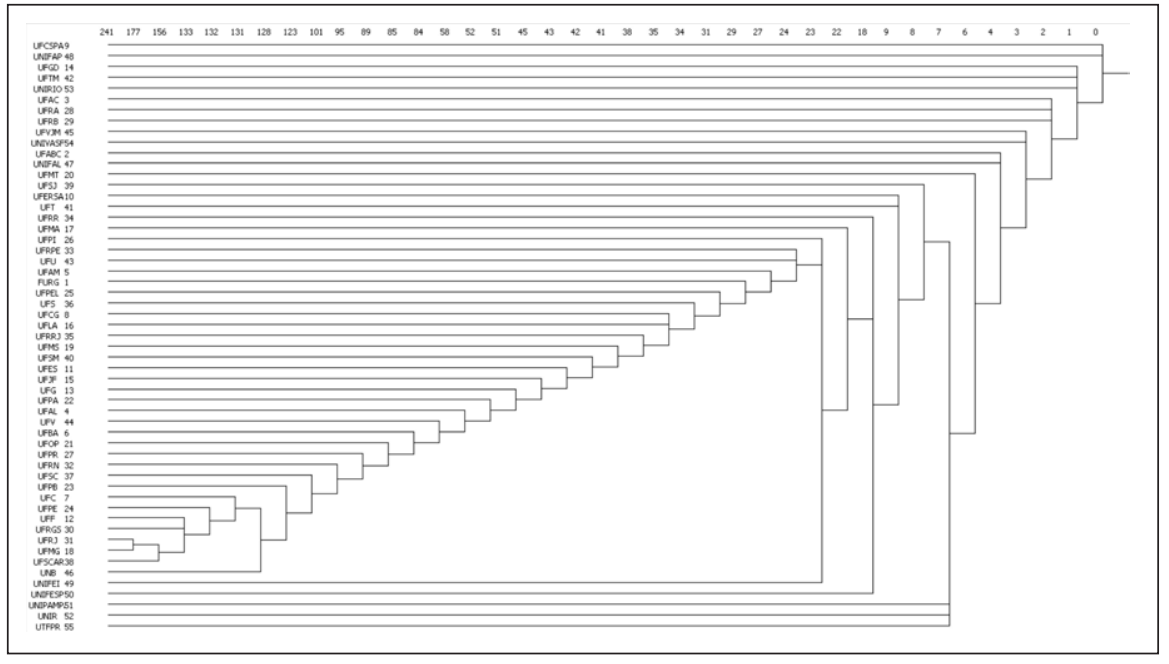

Figura 3. Cluster formados pelas Ifes

Para verificar quais desses nós proporcionam mais estabilidade à rede, ou seja, quais nós têm uma posição mais importante como colaboradores, foi necessário utilizar o algoritmo de conjuntos Lambda (ver Figura 4).

A configuração assumida pelos nós após a aplicação do algoritmo de Conjuntos Lambda coloca, assim como a representação gráfica de clusters, UFRJ e UFMG no primeiro nivel de agregação. Isso indica que, caso um desses nós fosse removido da rede, poderia haver uma obstrução, dificultando a possibilidade de colaboração entre as Ifes que formam clusters com as mesmas.

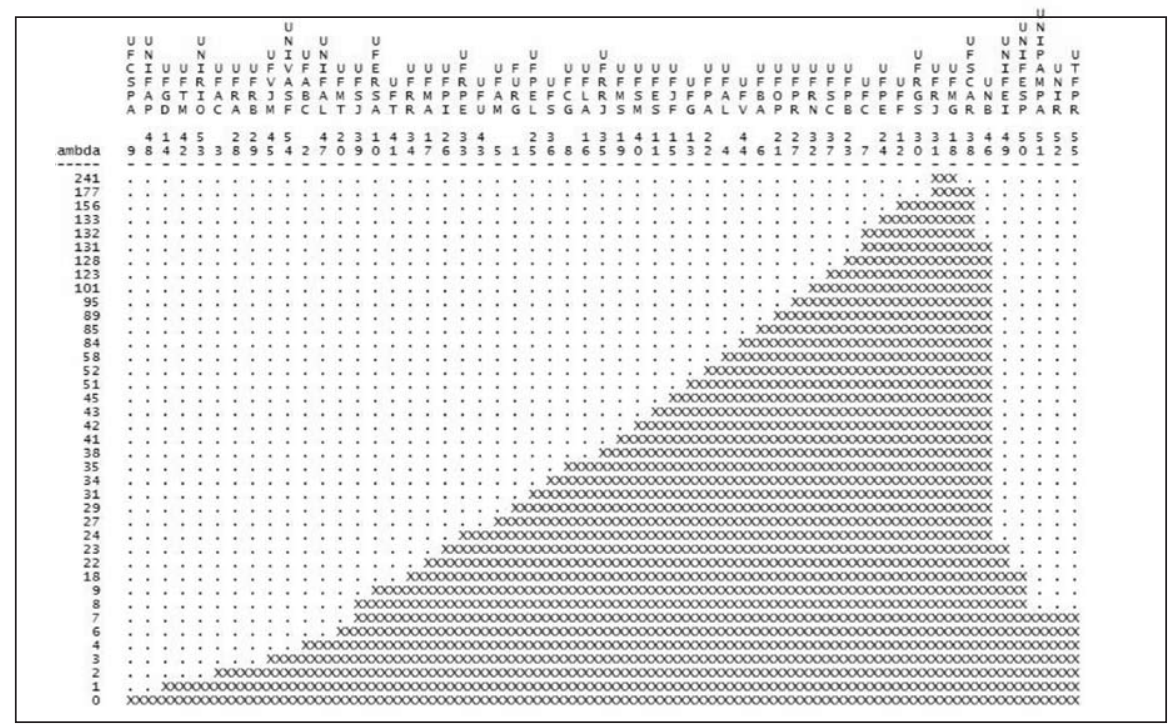

Figura 4. Conjunto Lambda formado pelas Ifes 
A proeminência relativa de um ator em relação a sua vizinhança (centralidade local) é dada pelo grau de centralidade, o qual, dada a baixa densidade da rede, foi de apenas 6,14\%. As 10 Ifes que se encontram na rede colaborativa de 1997-2007 em posição vantajosa por possuírem o maior número de conexões diretas/adjacentes são UFRJ, UFMG, UFRGS, UFPE, UFC, UnB, UFSCAR, Ufba, UFRN e UFF.

Cada universidade manteve conexões com 23,64 universidades. A dispersão dos fluxos foi alta, apesar do desvio padrão situar-se abaixo da média em 17,8.

A interação entre duas universidades não-adjacentes pode depender de duas outras universidades que se interpõem entre elas. 0 grau de intermediação admite que uma universidade esteja em posição favorável se ela faz parte de outras conexões que ligam outros pares de universidades na rede ou então se desempenha o papel de intermediária das interações entre esses atores. Observando-se a Figura 5, é possivel perceber que a universidade com maior grau de intermediação é a UFV, seguida da UFRJ, UFMG e UFSCAR na região Sudeste, UFPA na região Norte, UnB na região Centro-Oeste e UFC, UFPE, UFRPE, UFPB e UFRN na região Nordeste. Um aspecto importante que o grafo permite visualizar é que as Ifes da região Sudeste são as que apresentam o maior grau de intermediação, sendo que as Ifes citadas estão entre as 15 que apresentam maior produção nacional em números absolutos; mas esse fator, apesar de importante, não é determinante, já que a UFV $\left(11^{\mathrm{a}}\right)$ tem maior grau de intermediação, por exemplo, que a UFRJ que, entre as Ifes, é a que mais publicou artigos.

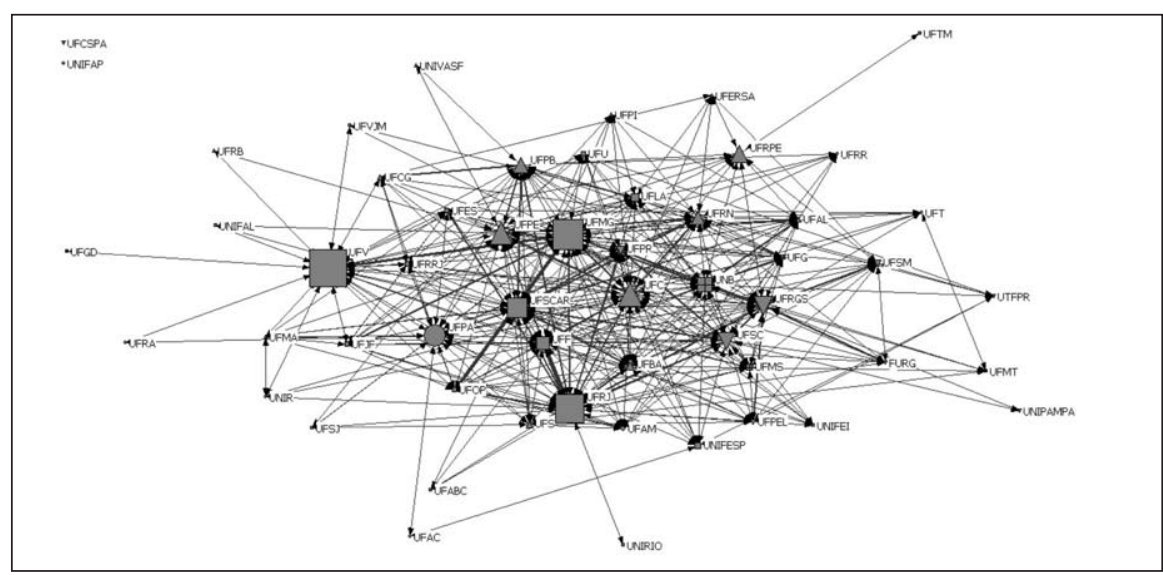

Figura 5. Visão geral da rede colaborativa de Ifes usando a métrica de intermediação 
Segundo a medida de proximidade, universidades que são capazes de alcançar outras universidades por meio de passos de comprimento mais curto, ou que dessa mesma forma podem ser alcançadas por outras universidades, encontram-se em posição favorável. Mas no caso da rede estudada é possivel perceber que essa medida não é decisiva quando aplicada ao grafo geral de todas as Ifes, pois, como a densidade da rede é muito baixa (algo em torno de $10 \%$, a distribuição das centralidades da rede não é uma medida conclusiva.

\section{Considerações finais}

Os resultados obtidos por meio das técnicas de análise de redes proporcionaram elementos que permitiram dar sentido aos dados empíricos. Assim, admitiu-se que a localização de determinada instituição tem um peso efetivo no caso da colaboração científica interinstitucional, porque ela só ocorre se a rede social a que pertence determinado nó propicia o conjunto de laços/conexões que permita levá-la a efeito. Cada uma das redes que integra a rede de colaboração de Ifes atua de acordo com lógicas próprias: pode-se falar em uma rede regional, interinstitucional, disciplinar, interdisciplinar, multidisciplinar etc. Daí, no caso específico dos autores colaboradores, cabe reconhecer que, além da esfera regida por laços de amizade, afinidade científica e profissional, também se agregam elementos/agentes de natureza conceitual, institucional e geográfica, que desempenham papel fundamental para o êxito da colaboração científica interdisciplinar.

Logo, não é a rede pessoal do ator que determina a colaboração, mas são as articulações estabelecidas por essa rede com outras redes sociais organizadas, consoante o princípio compartilhado de resolver problemas de cunho interdisciplinar, que tornam a colaboração provável. Assim, se, por exemplo, um autor ligado à área de Biologia não estiver inserido em uma rede colaborativa interinstitucional e se a rede pessoal dele não abrigar os laços que permitam acionar essa rede de colaboração, é pouco provável que a colaboração com outros campos científicos se dê; é preciso que o ator tenha os laços certos com os atores certos. 
Assim, a colaboração depende, no caso da rede de Ifes, do perfil dos laços da rede social de que toma parte o ator; depende da posição estrutural que os fluxos relacionais conferem a ele nessa rede social, isto é, a rede social de que o ator participa deve comportar vínculos que o inscrevam na rede de colaboração interinstitucional para efetivar a colaboração.

A perspectiva analítica trazida pelo fluxo máximo deixa ver que as universidades com o maior número de publicações possuem vínculos colaborativos análogos entre si e com as demais universidades da rede, ou seja, está à disposição dessas universidades o maior número de conexões diretas (alternativas) para alcançar as demais.

0 indicador de centralidade global registrou que a região Sudeste, representada pelas universidades UFMG, UFRJ, UFV e UFSCAR, em razão do número de conexões diretas estabelecido por essas universidades, ocupa posições centrais na rede, embora a UFC da região Nordeste também esteja em posição de destaque, até por ser a universidade com a maior produção entre as localizadas nessa região.

As medidas de centralidade demonstram a posição privilegiada, a importância de determinados nós na rede. Essa posição pode manifestar-se quando consideradas as relações entre os atores, de duas formas: influência e prestígio. Nesse caso, identificam-se os atores que constituem o foco de recepção dos vínculos relacionais; naquele, os atores que representam o centro desses vínculos. Assim, a alta centralidade, que as universidades do Sudeste apresentaram, permite inferir a influência, no tocante às colaborações, e o prestígio dessas universidades no âmbito nacional, no número de produção.

O recurso ao algoritmo Conjunto Lambda, utilizado para identificar os subgrupos de universidades que conferem mais estabilidade à rede, as universidades que tornam a distribuição de conexões colaborativas mais vulnerável à ruptura, pôs em evidência que universidades do Sul e Sudeste participaram dos níveis mais altos de agregação. Assim, as conexões que essas universidades estabeleceram entre si e com outras são de importância capital para garantir a estrutura das colaborações. 
Por conseguinte, esse conjunto de medidas que toma como referência a perspectiva das redes sociais põe em evidência um contexto reticular pouco propício à difusão de informação no âmbito nacional, uma vez que universidades de uma mesma região têm mais destaque na rede e fazem mais trocas entre si, quando se tem em conta a baixa conectividade das redes da baixa densidade e das grandes distâncias geodésicas entre as universidades. Assim, a seleção das universidades que constituem o resultado comum da aplicação das medidas de rede, fluxo máximo, centralidade, conjuntos Lambda e equivalência estrutural, faculta o reconhecimento da coincidência entre o proeminente papel desempenhado por UFRJ, UFMG, UFRGS e UFSCAR na rede de colaboração interna e a posição que ocuparam, em primeiro, segundo, terceiro e quarto lugar, respectivamente, na hierarquia das 10 universidades federais brasileiras com maior produção científica, dentro da amostra abordada.

Recebido em 31/07/2010. Aprovado em 05/10/2010.

\section{Referências bibliográficas}

BRUUN, H.; HUKKINEN, J.; HUUTONIEMI, K.; KLEIN, J. T. Promoting Interdisciplinary Research: The Case of the Academy of Finland. Helsinki: Academy of Finland, 2005. Disponivel em:<http:/ / www.aka.fi/Tiedostot/ Tiedostot/Julkaisut8_05\%20Promoting\%20Interdisciplinary\%20 Research_\% 20The\%20Case\%20of\%20the\%20Academy\%20of\%20Finland. pdf>. Acesso em: 07 out. 2008.

BURT, R. S. Structural holes: the social structure of competition. Cambridge: Harvard University, 1992.

HANNEMAN, R. Introduction to social network methods. Disponivel em: <http://www.researchmethods.org/NETTEXT.pdf>. Acesso em: 1 maio 2008.

KATZ, J. S.; MARTIN, B. R. What is the research collaboration? Research Policy, v. 26, n. 1, 1997, p. 1-35. Disponivel em: < http://www.sussex. ac.uk/Users/sylvank/pubs/Res_col9.pdf>. Acesso em: 16 abr. 2008. 
KATZ, J. S.; SMITH, D. Collaborative Approaches to Research. Final report for the HEFCE Fundamental Review of Research Policy and Funding. Leeds/Brighton: HEPU/SPRU, 2000. Disponivel em: < http:/ / www. hefce. ac.uk/research/review/consult/collc.doc>. Acesso em: 16 abr. 2008.

LEYDESDORFF, L.; ETZKOWITZ, H. Emergence of a triple helix of university industry government relations. Science and Public Policy, v. 23, n. 5,1996, p. $279-286$.

LEYDESDORFF, L.; ETZKOWITZ, H. The Triple Helix as a model for innovation studies. Science and Public Policy, v. 25, n. 3, 1998, p. 195203.

MEADOWS, A. J. A comunicação científica. Brasília: Briquet de Lemos, 1999.

MOODY, J. The Structure of a Social Science Collaboration Network: Disciplinary Cohesion from 1963 to 1999. American Sociological Review, v. 69, n. 2, 2004, p. 213-238.

NEWMAN, M. E. J. The structure of scientific collaboration networks. PNAS, v. 98, n. 2, 2001, p. 404-409.

SANCHO, R.; MORILLO, F.; DE FILIPPO, D.; GÓMEZ, l.; TERESA FERNÁNDEZ, M. T. Indicadores de colaboración científica intercentros en los países de América Latina. INCl, v. 31, n. 4, 2006, p. 284-292. Disponivel em: <http:// www.scielo.org.ve/scielo.php?script=sci_arttext\&pid=S037818442006000400008\&lng=es\&nrm=iso >. Acesso em: 16 fev. 2008.

SANTOS, P. D. Redes Colaborativas Interdisciplinares: um estudo cienciométrico de universidades federais brasileiras. Dissertação de Mestrado. Programa de Pós-Graduação em Engenharia da Informação, 2010, Universidade Federal do ABC (UFABC).

SONNENWALD, D. H. Scientific Collaboration: A Synthesis of Challenges and Strategies. Annual Review of Information Science and Technology, v. 4. Medford: Information Today, 2006. 